\title{
Treatment of Complex Cutaneous Leishmaniasis with Liposomal Amphotericin B
}

\author{
Maria Ubals 1,2,+(D), Pau Bosch-Nicolau ${ }^{3,+}+\mathbb{D}$, Adrián Sánchez-Montalvá ${ }^{3, *(\mathbb{D})}$, Fernando Salvador ${ }^{3}$, \\ Gloria Aparicio-Español 1,4, Elena Sulleiro ${ }^{5}\left(\mathbb{D}\right.$, Aroa Silgado ${ }^{5}$, Antoni Soriano-Arandes ${ }^{6}$ D, Maria Espiau 6 (D), \\ Berta Ferrer $^{7}$, Diana Pou ${ }^{8}$, Begoña Treviño ${ }^{8}$ (D) , Israel Molina ${ }^{3}$ and Vicente García-Patos ${ }^{1,4}$
}

1 Department of Dermatology, Hospital Universitari Vall d'Hebron, Universitat Autònoma de Barcelona, 08035 Barcelona, Spain; mariaubalscazorla@gmail.com (M.U.); glaparic74@gmail.com (G.A.-E.); vgarciapatos@gmail.com (V.G.-P.)

2 Doctoral Programme in Medicine and Translational Research: International Health Track, Facultat de Medicina, Universitat de Barcelona, 08036 Barcelona, Spain

3 Department of Infectious Diseases, Hospital Universitari Vall d'Hebron, PROSICS Barcelona, Universitat Autònoma de Barcelona, 08035 Barcelona, Spain; pau.boschnicolau@gmail.com (P.B.-N.); medinano@yahoo.es (F.S.); israelmolina@ymail.com (I.M.)

check for updates

Citation: Ubals, M.; Bosch-Nicolau, P.; Sánchez-Montalvá, A.; Salvador, F.; Aparicio-Español, G.; Sulleiro, E.; Silgado, A.; Soriano-Arandes, A.; Espiau, M.; Ferrer, B.; et al. Treatment of Complex Cutaneous Leishmaniasis with Liposomal Amphotericin B. Pathogens 2021, 10, 1253. https:// doi.org/10.3390/pathogens10101253

Academic Editors: André Luis Souza dos Santos, Koert Ritmeijer, Marta Helena Branquinha, Claudia Masini d'Avila-Levy, Cátia Lacerda Sodré and Igor de Almeida Rodrigues

Received: 5 August 2021

Accepted: 25 September 2021

Published: 28 September 2021

Publisher's Note: MDPI stays neutral with regard to jurisdictional claims in published maps and institutional affiliations.

Copyright: (c) 2021 by the authors. Licensee MDPI, Basel, Switzerland. This article is an open access article distributed under the terms and conditions of the Creative Commons Attribution (CC BY) license (https:/ / creativecommons.org/licenses/by/ $4.0 /)$.
4 Facultat de Medicina, Universitat Autònoma de Barcelona, 08035 Barcelona, Spain

5 Department of Clinical Microbiology, Hospital Universitari Vall d'Hebron, PROSICS Barcelona, Universitat Autònoma de Barcelona, 08035 Barcelona, Spain; esulleir@vhebron.net (E.S.); aroa.silgado@vhir.org (A.S.)

6 Department of Paediatrics, Hospital Universitari Vall d'Hebron, Universitat Autònoma de Barcelona, 08035 Barcelona, Spain; asoriano@vhebron.net (A.S.-A.); mespiau@vhebron.net (M.E.)

7 Department of Pathology, Hospital Universitari Vall d'Hebron, Universitat Autònoma de Barcelona, 08035 Barcelona, Spain; bferrer@vhebron.net

8 Tropical Medicine and International Health Unit, Drassanes-Vall d'Hebron, PROSICS Barcelona 08035 Barcelona, Spain; d.pou@vhebron.net (D.P.); btrevino.bcn.ics@gencat.cat (B.T.)

* Correspondence: adsanche@vhebron.net; Tel.: +34-93-274-6090; Fax: +34-93-489-40-91

+ These authors contributed equally to this work.

Abstract: Background: There is no consensus for the best treatment of complex cutaneous leishmaniasis (CL). We aimed to describe a cohort of CL, focusing on liposomal amphotericin B (L-AmB) treatment outcome. Methods: We performed a retrospective study in Vall d'Hebron University Hospital (Barcelona, Spain). All patients with parasitologically proven CL diagnosed from 2012 to 2018 were included. Results: The analysis included 41 patients with CL. The median age was 39 years (IQR 12- 66); 12 (29\%) were children, and 29 (71\%) were men. Regarding treatment, 24 (59\%) received local treatment, whereas $17(41 \%)$ had complex CL and were offered intravenous systemic treatment. Sixteen patients received L-AmB; eight (50\%) had adverse events, and three (19\%) discontinued treatment for safety reasons. All cases were considered cured within the first year post-treatment. Conclusions: L-AmB for complex CL showed no treatment failures, offering an alternative treatment option for patients with complex CL. Clinicians should pay close attention to the potential adverse events of L-AmB and adopt an active drug safety surveillance scheme to rapidly detect reversible side effects.

Keywords: complex cutaneous leishmaniasis; cutaneous leishmaniasis; liposomal amphotericin B; leishmania; systemic therapy

\section{Introduction}

Cutaneous leishmaniasis (CL) is a sand-fly-borne disease caused by 1 of 23 different pathogenic species of the genus Leishmania. The World Health Organization (WHO) estimates that one million cases of CL occur every year worldwide [1,2]. The protozoan parasites in promastigote form are transmitted to susceptible mammalian hosts by female sand flies (Phlebotomus, Lutzomyia). According to the geographic distribution, CL can be classified into New World CL (American Continent, transmitted by Lutzomyia sand 
flies) and Old World CL (Mediterranean basin, northeast Africa, Middle East, South Asia, transmitted by Phlebotomus sand flies). The most common species that cause New World CL are L. V (Viannia). brazilensis, L. V. panamensis and L.V. guayanensis; and Old World CL is generally caused by L. L (Leishmania). infantum, L. L. major and L. L. tropica. In the Mediterranean basin, the most common causative specie of CL is L. Infantum, and its usual reservoir is the dog. However, in recent years, an increasing incidence of other species has been observed due to returning travelers and migration movements (L. tropica, L. donovani and L. major) [3].

After a careful diagnostic evaluation, management should be individualized based on the classification in simple or complex CL. Complex CL is defined by a high risk of mucosal involvement, numerous or very large lesions, disseminated $\mathrm{CL}$, lesions located in places with difficult access to local treatment, immunosuppression or clinical failure of local therapy [4]. Patients with simple CL can be offered local therapy or observation without treatment. However, the Infectious Diseases Society of America (IDSA) guidelines recommend systemic treatment for patients with complex CL [4].

Systemic pentavalent antimonials $\left(\mathrm{Sb}^{\mathrm{v}}\right)$ have shown cure rates of $85-90 \%$ in studies on Old World CL and are currently considered the 'gold standard' comparator drug for evaluating the efficacy of new therapies [5]. However, this treatment is frequently associated with severe adverse events (i.e., cardiotoxicity, elevation of liver and pancreatic enzymes, and bone marrow suppression), and up to $25 \%$ of patients may discontinue treatment due to safety reasons [6]. Alternative systemic treatment options for complex $\mathrm{CL}$ are intravenous liposomal amphotericin B (L-AmB), intravenous or intramuscular pentamidine, oral miltefosine and oral azoles. Of them, only miltefosine has been granted FDA approval for this indication, based on recent clinical trials [7-9], although it is not commercially available in most European countries. Data regarding the efficacy and safety of L-AmB for Old World CL treatment are limited to scarce retrospective studies, although they showed promising efficacy results with cure rate up to $84 \%[10,11]$.

The scarcity of clinical information regarding systemic agents for the treatment of complex CL and heterogeneity of healing rates between studies challenges the assessment of the trade-off between the efficacy and toxicity of these treatments. In the case of L-AmB, for which no randomized controlled trials are available in the CL setting, data from the real-world use contribute to characterizing the patient profile that can benefit more from this treatment. In this study, we aim to characterize patients with CL treated in a tertiary hospital in Spain and assess the treatment outcomes in those receiving L-AmB according to the IDSA criteria for systemic treatment.

\section{Results}

\subsection{Demographic and Epidemiologic Characteristics}

Between 2012 to 2018, 41 patients were diagnosed with CL at Hospital Universitari Vall d'Hebron. The median age was 39 years (IQR 12-66), 12 (29\%) were children younger than 18 years, and $29(71 \%)$ were male. Sixteen patients (39\%) were migrants (13 from Morocco, 1 from Bolivia, 1 from Oman, and 1 from Honduras); fourteen (34\%) acquired the infection during an international trip (10 in Morocco, 2 in Thailand, 1 in Turkey, and 1 in Bolivia). Nine (22\%) patients, all autochthonous, had an immunosuppressing condition (6 anti-TNF $\alpha$ therapy recipients, 1 HIV infection, and 2 primary CD4 deficiency). Eighteen cases had no history of travel or immunosuppression. More data are shown in Table 1.

\subsection{Diagnostic Methods and Classification}

The median diagnostic delay was five months (IQR 2-8.25). Of the 32 cases analyzed for histopathology, $27(84 \%)$ had findings that confirmed CL diagnosis. Of the 38 samples tested using microbiology techniques, $34(89 \%)$ had a positive result (Table 1$)$. The etiological agent was studied in 34 of 41 confirmed cases (83\%): 21 (62\%) patients had L. infantum infection, and $9(26 \%)$ patients had L. major infection. The remaining $4(12 \%)$ had insufficient DNA for testing. 
Table 1. Baseline characteristics of study participants.

\begin{tabular}{|c|c|}
\hline COMPLETE CL COHORT DATA & $\mathrm{N}=41$ \\
\hline \multicolumn{2}{|l|}{ Demographic data } \\
\hline Age (years), median & 39 (IQR 12-66) \\
\hline Gender (male) & $29(70 \%)$ \\
\hline History of travel & $14(34 \%)$ \\
\hline Autochthonous infection & $25(61 \%)$ \\
\hline Immunosuppressing condition & $9(22 \%)$ \\
\hline \multicolumn{2}{|l|}{ Diagnostic method } \\
\hline Histopathological findings $(\mathrm{n}=32)$ & $27(84 \%)$ \\
\hline Positive PCR (n = 38) & $34(89 \%)$ \\
\hline \multicolumn{2}{|l|}{ Species identification $(\mathrm{n}=34)$} \\
\hline L. infantum & $21(62 \%)$ \\
\hline L. major & $9(26 \%)$ \\
\hline Indeterminate result & $4(12 \%)$ \\
\hline \multicolumn{2}{|l|}{ Type of CL } \\
\hline Simple CL & $24(59 \%)$ \\
\hline Complex CL & $17(41 \%)$ \\
\hline Immunocompromised & 9 \\
\hline Mucosal involvement & 2 \\
\hline Multifocal & 3 \\
\hline Large size & 3 \\
\hline L-AMB TREATMENT COHORT DATA & $\mathbf{N}=16$ \\
\hline \multicolumn{2}{|l|}{ Demographic data } \\
\hline Age (years), median & 44 (IQR 35-66) \\
\hline Gender (male) & $15(94 \%)$ \\
\hline History of travel & $6(38 \%)$ \\
\hline Autochthonous infection & $9(56 \%)$ \\
\hline Immunosuppressing condition & $8(50 \%)$ \\
\hline \multicolumn{2}{|l|}{ Diagnostic method } \\
\hline Histopathological findings $(\mathrm{n}=14)$ & $11(79 \%)$ \\
\hline Positive PCR $(\mathrm{n}=14)$ & $12(86 \%)$ \\
\hline \multicolumn{2}{|l|}{ Species identification } \\
\hline L. infantum & $7(44 \%)$ \\
\hline L. major & $5(31 \%)$ \\
\hline Unknown species & $4(25 \%)$ \\
\hline
\end{tabular}

CL: cutaneous leishmaniosis, IQR: interquartile range (25th and 75th percentile).

Twenty-four (59\%) patients presented simple CL, while 17 (41\%) patients had complex CL: 9 were immunocompromised, 2 had mucosal involvement, 3 multifocal disease, and 3 were large size ulcers. Lesions from some patients of the cohort are shown in Figure 1. Of the 16 patients with complex CL who received treatment with L-AmB, 7 had L. infan- 
tum infection, 5 L. major infection, and species identification could not be conducted in 4 patients.
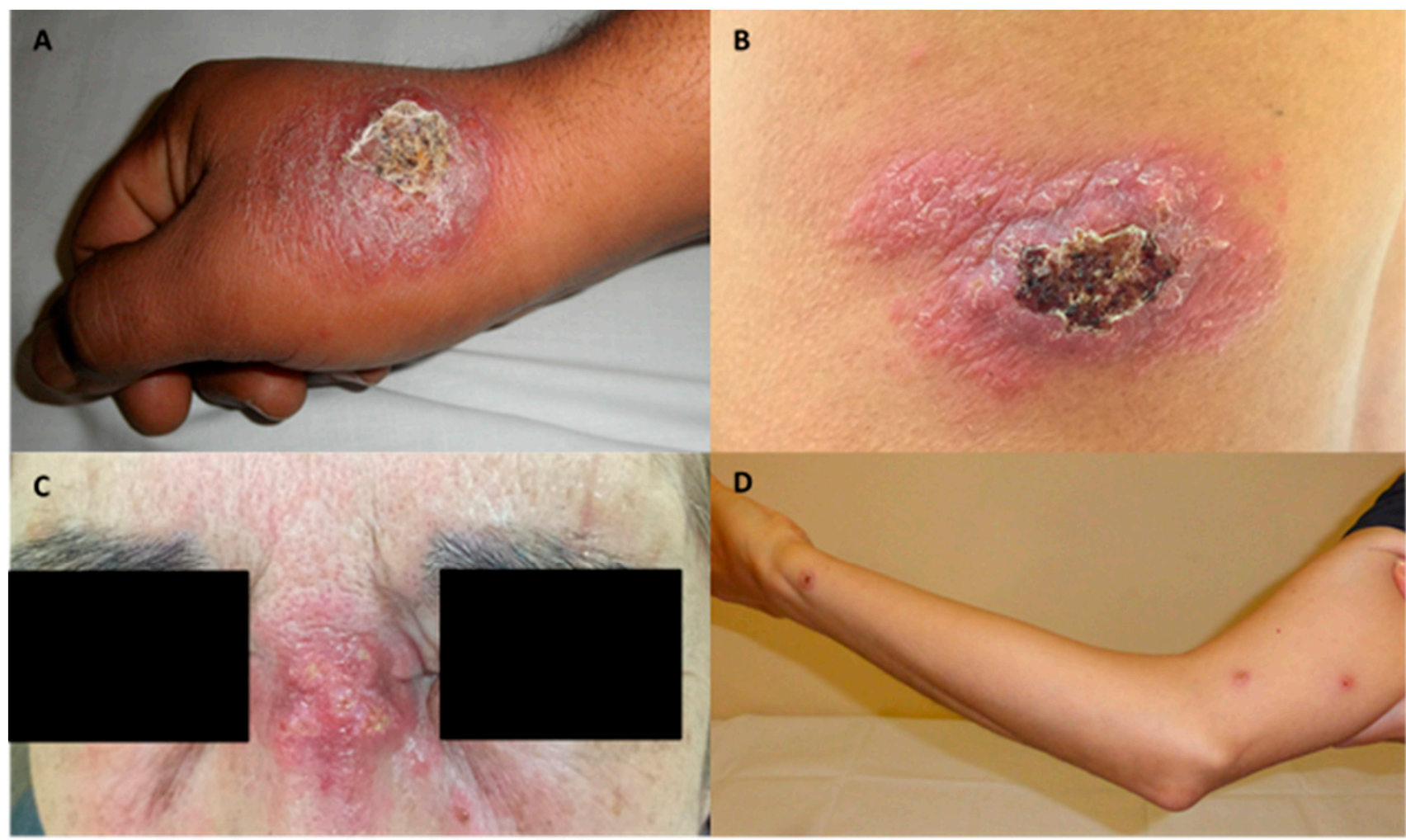

Figure 1. Images of four ulcers from different patients with complex features. (A): Hyperkeratotic and ulcerated plaque on the right hand from a patient with multifocal cutaneous leishmaniasis. (B): Hyperkeratotic 15 centimeters- plaque on the back. (C): Indurated plaque at the nasal root. (D): Subcutaneous nodules due to local dissemination on the right arm.

\subsection{Treatment Outcomes}

Patients with simple CL received local treatment (more information in Supplementary Table S1). Systemic therapy with L-AmB was initiated in 16 of 17 patients with complex CL: 13 completed the 5-day regimen, reaching a cumulative dose of $20 \mathrm{mg} / \mathrm{kg}$, and 3 had to discontinue treatment due to adverse events with at least a cumulative dose of $16 \mathrm{mg} / \mathrm{kg}$. All 16 were cured 3 months after treatment completion. All patients showed good progression, and no relapses were reported at 12 month follow-up.

One exceptional case with disseminated $C L$ in the context of visceral leishmaniasis (VL) was treated with pentamidine. It was administered in dosages of $4 \mathrm{mg} / \mathrm{kg} /$ day on alternate days for a total of 15 doses, not exceeding $300 \mathrm{mg}$ daily.

\subsection{Treatment Safety}

No serious adverse events were detected in the group of patients treated locally. However, $8(50 \%)$ of patients who received L-AmB had an adverse reaction (Table 2), all grade III or more, including 4 patients with acute kidney failure, 2 of them requiring hospital admission (detailed information of cases is depicted in Supplementary Table S2). All adverse events resolved after treatment discontinuation. Figure 2 shows the glomerular filtrate measured at three time points (before, immediately post-treatment, and 1 month after the therapy) in patients treated with L-AmB. The median decrease in the glomerular filtrate was $15.5 \mathrm{~mL} / \mathrm{min} / 1.73 \mathrm{~m}^{2}$ (IQR 10.5-27.75). All patients recovered normal kidney function three months after treatment. 
Table 2. Adverse events related to L-AmB treatment.

\begin{tabular}{cc}
\hline & $\mathbf{N}=\mathbf{1 6}$ \\
\hline Type of adverse events & $8(50 \%)$ \\
\hline $\begin{array}{c}\text { Infusional reaction } \\
\text { Acute kidney failure, glomerular filtrate } \\
(\mathrm{mL} / \mathrm{min})<50 \%\end{array}$ & $3(19 \%)$ \\
\hline Nausea/vomiting & $4(25 \%)$ \\
\hline Diarrhea & $4(25 \%)$ \\
\hline Fever & $1(6 \%)$ \\
\hline Severity of adverse events & $1(6 \%)$ \\
\hline Grade I-II & 0 \\
\hline Grade III & 6 \\
\hline Grade IV & 2 \\
\hline
\end{tabular}

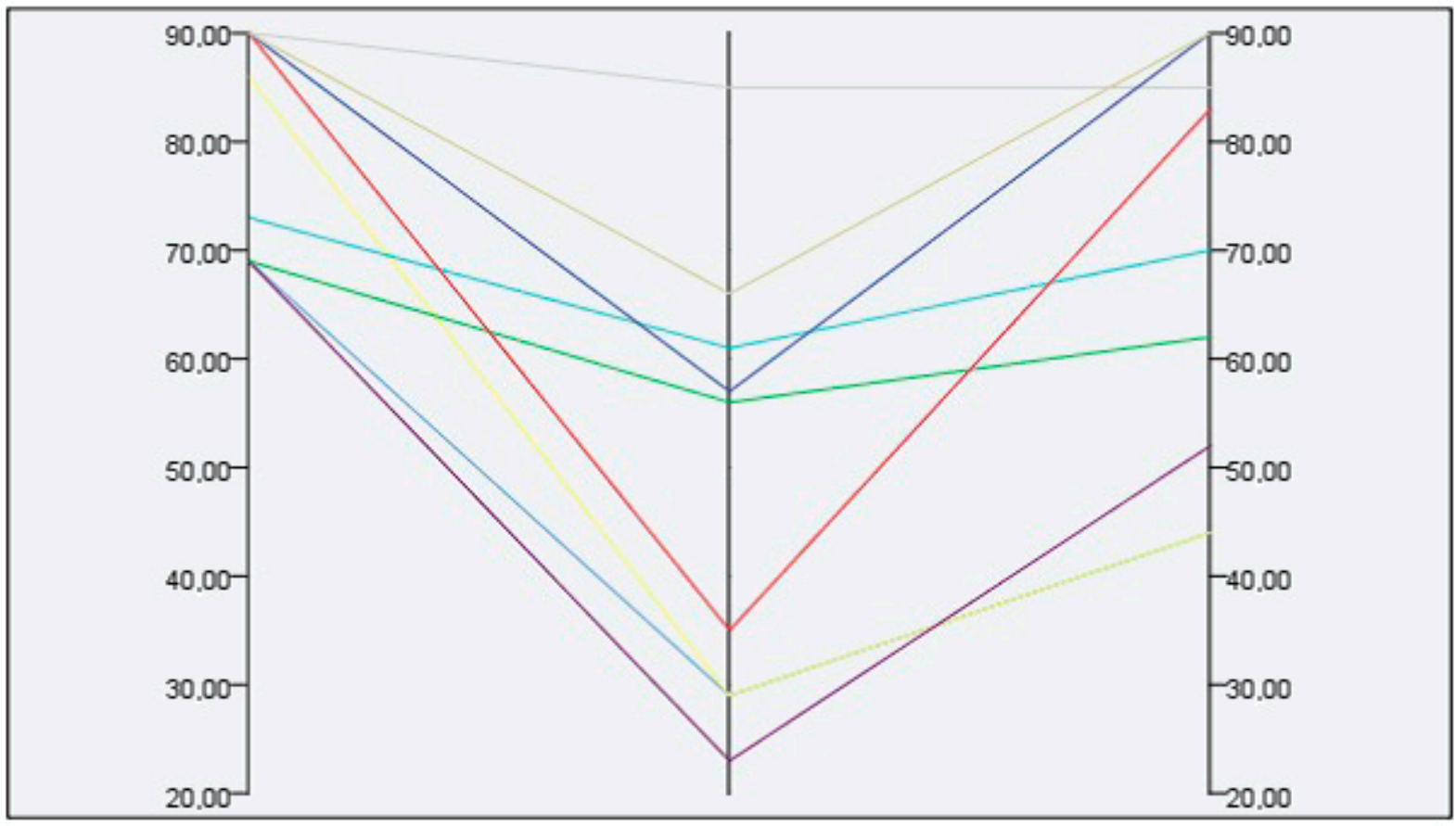

Figure 2. Kidney function test at different time points in patients treated with L-AmB. Glomerular filtrate $\left(\mathrm{mL} / \mathrm{min} / 1.73 \mathrm{~m}^{2}\right)$, time points-before treatment, immediately post-treatment and 1 month after treatment onset.

\section{Discussion}

In this cohort of 16 patients with complex CL, 5 day treatment with L-AmB was associated with good clinical outcomes, we observed complete clinical healing at 3 months, and lack of relapse at 12 months of follow-up. Species identification was in concordance with geographic exposure, with autochthonous $L$. infantum being the main species in our cohort $(62 \%)$. The high frequency of this species, more susceptible to L-AmB than others [10], may have contributed to the high healing rates observed in our cohort. Despite the high effectiveness of L-AmB in patients with complex CL, treatment resulted in high frequency of adverse events, either infusion-related events or nephrotoxicity.

Most CL self-heal over a period that may range from 2 to 18 months [12]; therefore, a conservative approach with local therapy should be appropriate in most CL infections, 
particularly those that are simple CL forms in immunocompetent hosts. However, patients with complex CL, such as the 16 cases reported in this analysis, may require systemic therapy to accelerate the healing of lesions, improve scarring and avoid the spread of the disease.

Like in many other neglected diseases, the poor quality of clinical evidence and lack of strong consensus on CL treatment [13,14] challenges making therapeutic decisions in the daily management of these patients. The main reasons for prescribing systemic treatment in our cohort included reducing scarring in large ulcers located in places with difficult access to local treatment, and immunosuppression. While this approach seems reasonable because it increases the delivery of the therapeutic agent to distant sites of potential spread, it is unclear to what extent all immunosuppressed patients would require systemic therapy. A recent review of 49 patients receiving therapy with TNF- $\alpha$ blockers $(57.1 \%$ and $10.2 \%$ with cutaneous and mucocutaneous leishmaniosis, respectively) showed positive outcomes after systemic therapy-mainly with L-AmB - and discontinuation of the TNF- $\alpha$ blocker therapy until clinical resolution [15]. However, the immune response against $L$. infantum is complex, and not all immunosuppressed patients might be at similar risk of complex CL. A careful evaluation of the accrued immunosuppression over time, mechanism of action of the immunosuppressant medication and consequence of removing the immunomodulation drugs helps to guide the management of these patients.

$\mathrm{Sb}^{\mathrm{v}}$ have been widely used for the treatment of complex CL. They have a high cure rate, but the treatment is long, with frequent adverse events ( $25 \%$ patients require treatment discontinuation), and regular clinical, cardiac and laboratory monitoring is mandatory [16]. $\mathrm{L}-\mathrm{AmB}$ and $\mathrm{Sb}^{\mathrm{v}}$ show comparable efficacy for treatment of complex CL [17]. However, L-AmB treatment is a 5 day course therapy, compared with the 3 week course treatment with $\mathrm{Sb}^{\mathrm{v}}$ drugs. A drawback with using L-AmB is its high cost, but the reduction in the number of follow-up visits, hospitalization days and the avoidance of blood tests and electrocardiograms required to monitory $\mathrm{Sb}^{\mathrm{v}}$ toxicity make $\mathrm{L}-\mathrm{AmB}$ cost beneficial in industrialized countries [18]. Miltefosine is an oral and well-tolerated therapy; however, clinical cure responses range from $50 \%$ to $90 \%$ depending on the species involved, and it is a still foreign unavailable medication in most European countries [7,19]. Lastly, Pentamidine is a second-line treatment, recommended when there is a therapeutic failure or in special situations, given its safety profile [5].

The main challenge of making therapeutic decisions regarding treatment with L$\mathrm{AmB}$ is the trade-off between the risk of CL progression and the toxicity associated with L-AmB. In our cohort, half of the patients experienced moderate toxicity events. The most common adverse events were acute infusion-related events, which are associated with rapid infusion of L-AmB [20] and may occur in a frequency of up to $20 \%$ [11,21], and nephrotoxicity, which has been observed in up to $45 \%$ of cases [11]. Adverse events are reversible after discontinuation of L-AmB and medical treatment with intravenous hydration. In our cohort, L-AmB was administered with intravenous hydration and was slowly infused in order to mitigate nephrotoxicity and acute-infusion reactions.

Our study is limited by the retrospective design and the small sample size, but it provides real-world information on complex CL treated with L-AmB in the Mediterranean basin, currently lacking in the literature. Taken together, our results confirm the effectiveness of L-AmB for treating complex CL. However, the frequency of adverse events such as nephrotoxicity in healthy patients as a consequence of treating a non-lethal condition raises important concerns. Our results highlight the need for carefully weighing the risks and benefits of this systemic therapy and considering the broad repertoire of local therapies [4] before initiating a systemic agent. Furthermore, there is an urgent need to provide clinicians with evidence-based information regarding the type of patient who is more suitable for systemic treatment with L-AmB and progress marketing of alternative systemic agents. 


\section{Materials and Methods}

\subsection{Patients' Selection and Data Collection}

We retrospectively analyzed all cases of microbiologically proven CL from January 2012 to December 2018 diagnosed at Hospital Universitari Vall d'Hebron (Barcelona, Spain).

The diagnosis of CL was based on clinical suspicion, with laboratory confirmation by either histopathology findings compatible with amastigotes and/or the presence of molecular test positive for Leishmania spp. analyzed with real-time polymerase chain reaction (PCR). A duplex real-time PCR technique was performed following the protocol described by Mary et al. [22] with some modifications. The protocol includes two targets, a specific region of leishmania spp. Kinetoplast DNA and human RNase P gene as internal control. A sample was considered positive for Leishmania DNA when the threshold cycle (Ct) for the Leishmania target was $<40$ and negative when did not detect the specific target but detected internal amplification.

We reviewed electronic health records to gather demographic and clinical information of the patients, including age, gender, country of origin, history of immunosuppressive conditions, concomitant medication, travel history, lesion size and location, diagnostic methods, treatment received, adverse events and outcome.

\subsection{Treatment Criteria}

According to the hospital procedures, systemic treatment with L-AmB (Ambisome®, Gilead Sciences, Inc, Madrid, Spain) was offered based on the presence of multiple lesions $(\geq 5)$, large individual skin lesion $(\geq 5 \mathrm{~cm})$, local dissemination (satellite lesions, large lymphadenopathy or subcutaneous nodules), CL caused by Leishmania species associated with increased risk for mucosal leishmaniasis, and lesion on the face, joints or genitalia (places with difficult access to local treatment). Additionally, immunocompromised hosts or patients with treatment failure to local therapy were offered systemic treatment [4]. L-AmB is an approved course treatment in Spain for VL and fungal infections; the physicians prescribed L-AmB off-label as part of a compassionate use for complex CL treatment. L-AmB was given daily for five consecutive days at a dose of $4 \mathrm{mg} / \mathrm{kg}$ /day in a $500 \mathrm{~mL}$ glucose $5 \%$ and infused over 3-4 h. The total cumulative dose for each patient was $20 \mathrm{mg} / \mathrm{kg}$. Active safety monitoring assessment protocols in place included blood cell count and basic biochemistry tests before and after treatment completion.

\subsection{Outcome Criteria}

Based on hospital protocols, patients with CL were followed for 12 months after treatment (twice a month until re-epithelialization and every three months thereafter). The clinical outcome was assessed at three months after treatment and classified as (1) healing (the ulcer was completely re-epithelialized), (2) partial response ( $\geq 50 \%$ decrease in the largest diameter of the main ulcer), and (3) treatment failure ( $<50 \%$ decrease in the largest diameter of the main ulcer). Relapse was defined as a deterioration of skin lesion after a period of improvement within the 12 months after treatment completion. The relapse-free cure at 12 months was chosen as complementary endpoint.

\subsection{Statistical Analysis}

We performed a descriptive analysis regarding epidemiological data, clinical manifestations and treatment outcomes. Qualitative variables were defined as absolute number and percentages and quantitative as the median and interquartile range (IQR), defined as the 25 th and 75 th percentiles. No hypothesis tests were performed.

\subsection{Ethics Statement or Compliance with Ethical Standards}

The study protocol was approved by the Ethical Review Boards of Hospital Universitari Vall d'Hebron (Barcelona, Spain), approval number: EOM(AG)016/2021(5809). An exemption from obtaining informed consent was granted. Procedures were performed in 
accordance with the ethical standards laid down in the Declaration of Helsinki as revised in 2013.

\section{Conclusions}

Our experience shows high cure rates for complex CL with systemic L-AmB. Although adverse events are common, they are reversible and can be anticipated with an active safety monitoring assessment strategy. Considering the scarcity of therapeutic options for complex $\mathrm{CL}, \mathrm{L}-\mathrm{AmB}$ is currently convenient in a selected group of patients with complex CL when the benefit outweighs the risks because the course is short and tolerable. However, further research with alternative drugs is urgently needed.

Supplementary Materials: The following are available online at https://www.mdpi.com/article/10 .3390/ pathogens10101253/s1, Table S1: Local treatment and evolution of simple CL cases, Table S2: Age, comorbidities, concomitant medication, type and grade of adverse events presented in the cohort of complex CL treated with L-AmB.

Author Contributions: Conceptualization, M.U., A.S.-M., F.S., G.A.-E., A.S.-A. and V.G.-P.; methodology, M.U., P.B.-N., G.A.-E., A.S.-M., A.S., A.S.-A., M.E. and F.S.; software, M.U. and A.S.-M.; validation, M.U., G.A.-E., A.S.-M., F.S., A.S.-A. and V.G.-P.; formal analysis, M.U., A.S. and A.S.-M.; investigation, M.U., P.B.-N., G.A.-E., A.S.-M., A.S., A.S.-A., M.E. and F.S.; resources, V.G.-P. and I.M.; data curation, M.U. and A.S.-M.; writing-original draft preparation, M.U.; writing-review and editing, P.B.-N., A.S.-M., F.S., E.S., A.S.-A. and V.G.-P.; visualization, G.A.-E., E.S., A.S.-M., B.F., D.P., B.T. and I.M.; supervision, G.A.-E., E.S., B.F., V.G.-P., I.M., A.S.-M., F.S. and A.S.-A.; project administration, I.M. and V.G.-P.; funding acquisition, V.G.-P. and I.M. All authors have read and agreed to the published version of the manuscript.

Funding: This research received no external funding.

Institutional Review Board Statement: The study protocol was approved by the Ethical Review Boards of Hospital Universitari Vall d'Hebron (Barcelona, Spain).

Informed Consent Statement: An exemption from obtaining informed consents was granted.

Data Availability Statement: The data presented in this study are available on request. The data are not publicly available due to privacy.

Acknowledgments: The authors would like to thank Oriol Mitjà and Gerard Carot-Sans for his thorough review of the final version of the manuscript and the members of the skin NTDs unit of the Hospital Germans Trias i Pujol for their valuable and insightful comments on the study results. Adrián Sánchez- Montalvá was supported by a postdoctoral grant “Juan Rodés" (JE18/00022) from Instituto de Salud Carlos III through the Ministry of economy and competitiveness, Spain.

Conflicts of Interest: The authors declare no conflict of interest.

\section{References}

1. Alvar, J.; Vélez, I.D.; Bern, C.; Herrero, M.; Desjeux, P.; Cano, J.; Jannin, J.; Boer, M.D.; the WHO Leishmaniasis Control Team. Leishmaniasis Worldwide and Global Estimates of Its Incidence. PLoS ONE 2012, 7, e35671. [CrossRef] [PubMed]

2. Goto, H.; Lindoso, J.A.L. Cutaneous and Mucocutaneous Leishmaniasis. Infect. Dis. Clin. N. Am. 2012, 26, 293-307. [CrossRef] [PubMed]

3. Ready, P.D. Leishmaniasis emergence in Europe. Eurosurveillance 2010, 15, 29-39. [CrossRef]

4. Aronson, N.; Herwaldt, B.L.; Libman, M.; Pearson, R.; Lopez-Velez, R.; Weina, P.; Carvalho, E.; Ephros, M.; Jeronimo, S.; Magill, A. Diagnosis and Treatment of Leishmaniasis: Clinical Practice Guidelines by the Infectious Diseases Society of America (IDSA) and the American Society of Tropical Medicine and Hygiene (ASTMH). Am. J. Trop. Med. Hyg. 2016, 96, 24-45. [CrossRef] [PubMed]

5. Chakravarty, J.; Sundar, S. Current and emerging medications for the treatment of leishmaniasis. Expert Opin. Pharmacother. 2019, 20, 1251-1265. [CrossRef]

6. Oliveira, L.F.; Schubach, A.O.; Martins, M.M.; Passos, S.; Oliveira, R.D.V.C.D.; Marzochi, M.C.; Andrade, C.A. Systematic review of the adverse effects of cutaneous leishmaniasis treatment in the New World. Acta Trop. 2011, 118, 87-96. [CrossRef]

7. Machado, P.R.; Ampuero, J.; Guimarães, L.H.; Villasboas, L.; Rocha, A.T.; Schriefer, A.; Sousa, R.S.; Talhari, A.; Penna, G.; Carvalho, E.M. Miltefosine in the Treatment of Cutaneous Leishmaniasis Caused by Leishmania braziliensis in Brazil: A Randomized and Controlled Trial. PLoS Neglected Trop. Dis. 2010, 4, e912. [CrossRef]

8. Soto, J.; Toledo, J.T. Oral miltefosine to treat new world cutaneous leishmaniasis. Lancet Infect. Dis. 2007, 7, 7. [CrossRef] 
9. Van Thiel, P.P.A.M.; Leenstra, T.; Kager, P.A.; De Vries, H.J.; Van Vugt, M.; Van Der Meide, W.F.; Bart, A.; Zeegelaar, J.E.; Van der Sluis, A.; Schallig, H.D.F.H.; et al. Miltefosine treatment of Leishmania major infection: An observational study involving dutch military personnel returning from northern Afghanistan. Clin. Infect. Dis. 2010, 50, 80-83. [CrossRef]

10. Guery, R.; Henry, B.; Martin-Blondel, G.; Rouzaud, C.; Cordoliani, F.; Harms, G.; Gangneux, J.-P.; Foulet, F.; Bourrat, E.; Baccard, M.; et al. Liposomal amphotericin B in travelers with cutaneous and muco-cutaneous leishmaniasis: Not a panacea. PLoS Neglected Trop. Dis. 2017, 11, e0006094. [CrossRef]

11. Wortmann, G.; Pierson, J.; Weintrob, A.; Zapor, M.; Magill, A.; Ressner, R.; Fraser, S.; Hartzell, J. Lipsosomal Amphotericin B for Treatment of Cutaneous Leishmaniasis. Am. J. Trop. Med. Hyg. 2010, 83, 1028-1033. [CrossRef]

12. Scott, P.; Novais, F.O. Cutaneous leishmaniasis: Immune responses in protection and pathogenesis. Nat. Rev. Immunol. 2016, 16, 581-592. [CrossRef]

13. Heras-Mosteiro, J.; Monge-Maillo, B.; Pinart, M.; Lopez Pereira, P.; Reveiz, L.; Garcia-Carrasco, E.; Campuzano Cuadrado, P.; Royuela, A.; Mendez- Roman, I.; López- Vélez, R. Interventions for Old World cutaneous leishmaniasis. Cochrane Database Syst. Rev. 2017, 12.

14. Reveiz, L.; Maia-Elkhoury, A.N.S.; Nicholls, R.S.; Romero, G.; Yadon, Z.E. Interventions for American Cutaneous and Mucocutaneous Leishmaniasis: A Systematic Review Update. PLoS ONE 2013, 8, e61843. [CrossRef]

15. Bosch-Nicolau, P.; Ubals, M.; Salvador, F.; Sánchez-Montalvá, A.; Aparicio, G.; Erra, A.; De Salazar, P.M.; Sulleiro, E.; Molina, I. Leishmaniasis and tumor necrosis factor alpha antagonists in the Mediterranean basin. A switch in clinical expression. PLoS Neglected Trop. Dis. 2019, 13, e0007708. [CrossRef]

16. Monge-Maillo, B.; López-Vélez, R. Therapeutic Options for Old World Cutaneous Leishmaniasis and New World Cutaneous and Mucocutaneous Leishmaniasis. Drugs 2013, 73, 1889-1920. [CrossRef]

17. Senchyna, A.; Simon, S.; Cissé, H.; Ginouves, M.; Prevot, G.; Alcoba, G.; Demar, M.P.; Couppie, P.; Blaizot, R. American cutaneous leishmaniasis in French Guiana: A retrospective comparison between liposomal amphotericin B and meglumine antimoniate. Br. J. Dermatol. 2020, 183, 389-391. [CrossRef] [PubMed]

18. Mistro, S.; Gomes, B.; Rosa, L.; Miranda, L.; Camargo, M.; Badaró, R. Cost-effectiveness of liposomal amphotericin B in hospitalised patients with mucocutaneous leishmaniasis. Trop. Med. Int. Health 2017, 22, 1569-1578. [CrossRef] [PubMed]

19. Mosimann, V.; Blazek, C.; Grob, H.; Chaney, M.; Neumayr, A.; Blum, J. Miltefosine for Mucosal and Complicated Cutaneous Old World Leishmaniasis: A Case Series and Review of the Literature. Open Forum Infect. Dis. 2016, 3, ofw008. [CrossRef] [PubMed]

20. Sau, K.; Mambula, S.S.; Latz, E.; Henneke, P.; Golenbock, D.T.; Levitz, S.M. The Antifungal Drug Amphotericin B Promotes Inflammatory Cytokine Release by a Toll-like Receptor- and CD14-dependent Mechanism. J. Biol. Chem. 2003, 278, 37561-37568. [CrossRef] [PubMed]

21. Shirzadi, M.R. Lipsosomal amphotericin B: A review of its properties, function, and use for treatment of cutaneous leishmaniasis. Res. Rep. Trop. Med. 2019, 10, 11-18. [CrossRef] [PubMed]

22. Mary, C.; Faraut, F.; Lascombe, L.; Dumon, H. Quantification of Leishmania infantum DNA by a Real-Time PCR Assay with High Sensitivity. J. Clin. Microbiol. 2004, 42, 5249-5255. [CrossRef] [PubMed] 